\title{
Principle of an acoustical method for estimating the centroid position of the earcanal wall normal velocity induced by bone-conducted stimulation: Numerical evaluation
}

Kévin Carillo

École de technologie supérieure, Montréal, Québec, Canada

email: kevin.carillo.1@ens.etsmtl.ca

Olivier Doutres

École de technologie supérieure, Montréal, Québec, Canada

Franck Sgard

IRSST, Montréal, Québec, Canada

(Date : June 07, 2021)

Authors' accepted manuscript

Article published in Applied Acoustics, Volume 182, November 2021, 108245

The final publication is available at https://doi.org/10.1016/j.apacoust.2021.108245

(C) 2021. This manuscript version is made available under the CC-BY-NC-ND 4.0 license

http://creativecommons.org/licenses/by-nc-nd/4.0/ 
1 Abstract: The occlusion effect is commonly experienced as the altered perception of one's own

2 physiological noise when the earcanal entrance is blocked. Objectively, this phenomenon corresponds

3 to an acoustic pressure increase in the occluded earcanal. The occlusion effect originates from the

4 earcanal wall normal vibration and depends on the spatial distribution of the latter. At low frequencies,

5 this spatial distribution can be characterized by the position of its centroid along the earcanal middle

6 axis. This paper describes the principle of an acoustical method for estimating this centroid position at

7 low frequencies. The proposed method consists in measuring the eardrum acoustic pressure transfer

8 function between the earcanal open and occluded by an external capped duct coupled to the earcanal

9 entrance of a subject submitted to a bone-conducted stimulation. The centroid position is then

10 estimated at the antiresonance frequency of the coupled system using an associated electro-acoustic

11 model. The proposed method is evaluated and investigated numerically using a 3D finite element

12 model of an outer ear. The sensitivity of the method is shown to increase with frequency. To maximize

13 the method accuracy, the radius of the coupling duct must be as large as possible (in the limits of the

14 earcanal entrance dimension) and any incomplete seal between the duct and the earcanal entrance must

15 be avoided. Also, the coupling position of the duct and its temperature must be known as precisely as

16 possible. On the contrary, the proposed method does not require the knowledge of the eardrum acoustic 17 impedance.

18 Keywords: outer ear, acoustic antiresonance, earcanal wall vibration, electro-acoustic model, finite

19 element model 


\section{Introduction}

21 The occlusion effect (OE) is usually described as the increased perception of the bone-conducted 22 part of one's own physiological noise (e.g., one's own voice, chewing, heartbeat, etc.) when the earcanal (EC) entrance is covered or blocked. This phenomenon is most significant at low frequencies, typically below $1 \mathrm{kHz}$, and decreases above [1]. The OE contributes to the lack of comfort associated with wearing hearing protection devices, in particular for earplugs whereas earmuffs are less concerned, and partly explains their non-use [2]. Hearing aid users can also be affected by the OE [3], which is, however, greatly reduced by using vents or open-fit hearing aids [4]. Bone-conducted sound propagates into the body from the excitation source (e.g., vocal cords, bone-transducer, etc.) to the basilar membrane of the cochlea in the inner ear, which results in a hearing sensation. The hearing by bone-conduction has been extensively investigated since the past century and several sound transmission pathways have been highlighted through the outer ear, the middle ear and the inner ear itself [5] [6] [7] [8] [9] [10] [11] [12] [13] [14]. The occlusion of the EC entrance alters the contribution of the outer ear pathway to the hearing by bone-conduction (mainly at low frequencies below $1 \mathrm{kHz}$ ). This is due to the increased acoustic pressure generated by the EC wall when the EC entrance is occluded. The vibration of the EC wall constitutes the source of the OE.

This vibration is expected to be maximum in the cartilaginous part of the EC and minimum in the bony part where tissues are less likely to vibrate [10]. Most probably because of experimental difficulty, the spatial distribution of the EC wall normal vibration has never been directly measured. Indeed, direct measurement methods such as Doppler laser vibrometer or accelerometer could be very intricate to perform in a human EC due to its small size, its tortuosity and the presence of hair and wax. However, the spatial distribution of the EC wall normal vibration has significant influence on the vibroacoustic behavior of the open EC [15]. In addition, this distribution governs the reduction of the OE 
with insertion depth [16] [17]. Furthermore, the EC wall vibration distribution could influence the

contribution of earplugs to the OE [18] [19] [20]. Also, this distribution could be influenced by the bone-conducted stimulation which is known to influence the OE [18] [21] [22] but remains unexplained. The EC wall normal vibration distribution is therefore of great interest to study the boneconduction outer ear pathway and the OE.

Using a 3D finite element (FE) model of an outer ear, the authors have proposed to characterize the spatial distribution of the EC wall normal vibration by its centroid position [15]. This centroid position represents the center of normal velocity distributed over the EC wall surface. Also, the authors have shown that the centroid position corresponds to the location of the equivalent volume velocity source representing the EC wall normal vibration in an associated electro-acoustic (EA) model of the open EC. Therefore, the centroid position could be used as a vibratory indicator of the EC wall normal vibration distribution to study its parameters of influence (e.g., bone-conducted stimulation and position, anatomical differences, etc.). In addition, the knowledge of the centroid position could possibly guide to the optimal insertion depth of in-ear devices to reduce the OE without inserting them into the bony part of the EC.

In this paper, an indirect method is proposed to estimate this centroid position at low frequencies. This method is inspired from an experiment made by Huizing in 1960 [8]. In order to highlight a standing wave pattern in the occluded EC, Huizing measured the objective OE (i.e., sound pressure level (SPL) difference between occluded and open EC) induced by an external capped duct coupled to the EC entrance of a human subject submitted to a bone-conducted stimulation. Negative OEs were observed depending on the length of the coupling duct. Huizing interpreted these minima as acoustic resonances of the coupled system and assumed the occurrence of an acoustic pressure minimum at the eardrum [8]. In this paper, however, it is shown that the acoustic pressure node does not occur at the 
eardrum but at the centroid position of the EC wall normal velocity induced by the bone-conducted

67 stimulation. Using an EA model of the EC coupled to the external duct, the centroid position can be estimated from the lowest antiresonance frequency of the coupled system. This paper presents the experimental setup, the measurement procedure and the EA model associated with the indirect method.

Then, the method is evaluated and investigated numerically using a 3D FE model of an outer ear open and occluded by an external duct. Finally, the influence of several parameters of the coupled system on the estimation provided by the method is studied. The knowledge of the parameters influencing the method is of great interest for a future experimental application on human subjects.

\section{4}

75

76

77

\section{Principle of the method}

To estimate the centroid position of the EC wall normal velocity, the proposed indirect method requires (i) the physical measurement of the acoustic pressure transfer function between the EC open and occluded by an external capped duct coupled at the EC entrance and (ii) analysis using an EA model of the EC coupled to the same duct. Section 2.1 describes the experimental setup and the measurement procedure of the proposed indirect method. Then, Sec. 2.2 details the EA model associated with the indirect method and used to estimate the centroid position of the EC wall normal velocity. Finally, Sec. 2.3 presents the methodology of the numerical evaluation of the proposed method using a 3D FE model of an outer ear. No measurements are performed in this work. The experimental evaluation of the proposed method will be the subject of a future paper.

\subsection{Experimental setup and measurement procedure}

In both open and occluded ear, the acoustic pressure is measured close to the eardrum of a human subject submitted to a bone-conducted stimulation (e.g., bone-transducer applied to the forehead or the mastoid [21]). Figure 1 illustrates the corresponding experimental setup. In the occluded case (see Figure 1 (b)), a circular cross-section coupling duct is used. Its outer cross-sectional area must fit in the 
EC entrance plane. Since the EC cross-sections (including the EC entrance) are generally not circular

90 [23], the circular cross-section of the coupling duct is necessarily smaller than the EC entrance surface.

91 The coupling duct is not inserted in the EC but rather held in position in the concha using a "duct

92 holder". The duct holder could consist of an imprint of the concha up to the EC entrance plane and

93 including a hole in which the coupling duct would be inserted. In practice, determining the position of

94 the acoustic entrance of the EC is not trivial [24] [25]. Therefore, the EC entrance used in the proposed

95 method could be chosen based on geometrical consideration [26]. In any case, however, the proposed

96 method can only estimate the centroid position of the EC wall normal velocity between the chosen EC

97 entrance plane and the eardrum as explained in the following.

The lowest antiresonance frequency of the coupled system is identified from the acoustic pressure transfer function and corresponds to the first local minimum associated to a sudden phase shift (see Sec. 3.1). An EA model of the coupled system (presented in next section) is then used to estimate the

101 centroid position of the EC wall normal velocity. The estimation consists in minimizing the difference between the experimental and the simulated antiresonance frequency by varying both frequency and source position in the EA model. The latter requires the knowledge of the EC shape function between

104 the eardrum and the coupling duct and the geometric dimensions (inner radius and length) of the 105 coupling duct. The EC shape can be obtained from CT or MRI images [27], 3D scans [28], EC imprint

106 [26] or estimated using indirect acoustic method [29]. In theory, the EA model also requires the 107 tympanic membrane acoustic impedance. 

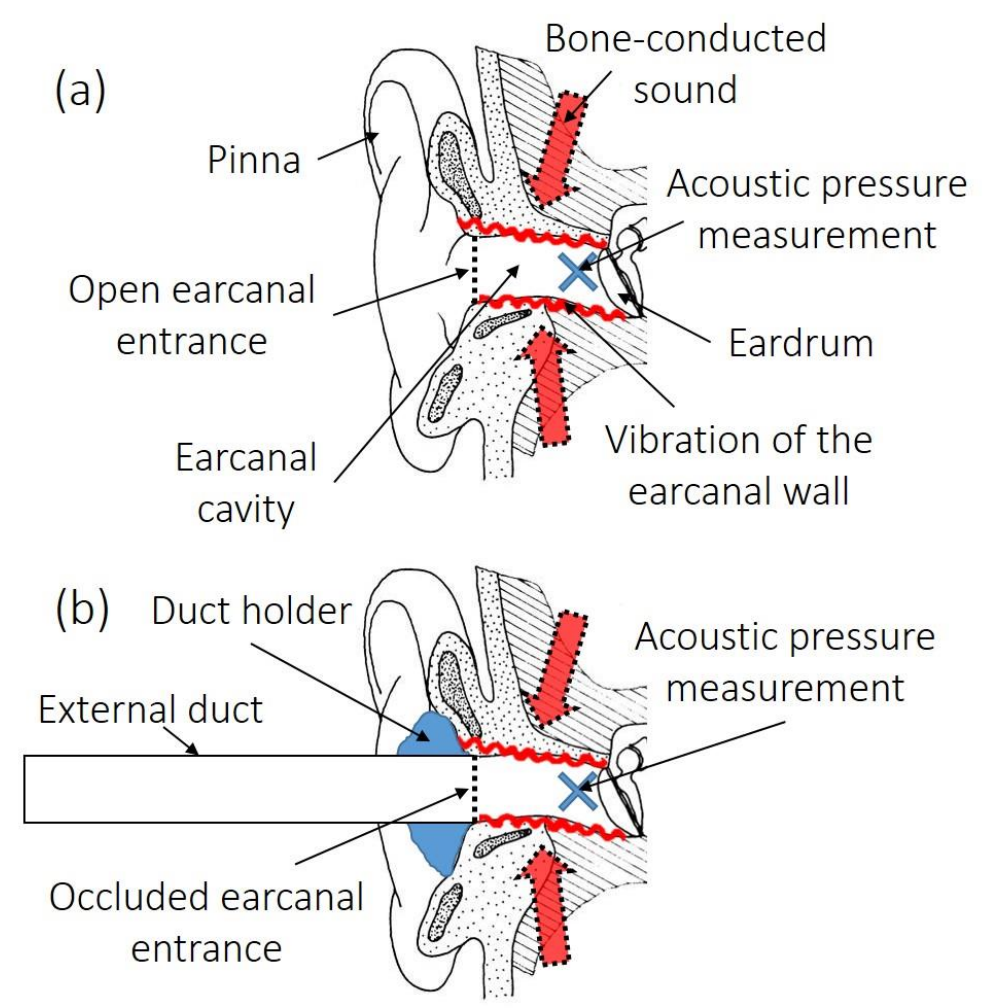

Figure 1: (Color online) (a) Open and (b) occluded EC experimental setup associated with the indirect method proposed to estimate the centroid position of the EC wall normal vibration induced by a boneconducted stimulation.

\section{$108 \quad 2.2$ Electro-acoustic model associated with the proposed method}

109 This section presents the EA model used in the proposed indirect method to estimate the centroid 110 position of the EC wall normal velocity. This model is illustrated in Figure 2 and consists of the EC 111 coupled to an external duct. This model is adapted from a previous model developed by the authors

112 [15]. The EC is considered as a circular cross-sectional duct of curvilinear length $l_{E C}$ and radius $r_{E C}(z)$

113 where $z$ is the EC middle axis. These parameters are calculated from the measured EC shape [23]. The 114 equivalent acoustic source Q represents the vibrating EC wall and is located at a position $l_{c}$ which

115 corresponds to the centroid position of the EC wall normal velocity [15]. The EC downstream section 116 goes from the eardrum to the source while the upstream section goes from the source to the EC 117 entrance. An acoustic impedance $\hat{Z}_{T M}$ is defined at the eardrum. At low frequencies, the downstream 
118 section is governed by its compressibility effect of acoustic compliance $C_{d}$, associated with acoustic

119 resistance $R_{d}$. The vibro-acoustic behavior of the EC upstream section depends on its inertia and 120 compressibility effects through the acoustic mass $L_{u}$ and acoustic compliance $C_{u}$ and their associated 121 resistances $R_{u, v i}$ and $R_{u, t h}$. Calculation of these constants is detailed in [15]. The vibro-acoustic

122 behavior of the EC upstream section also depends on the acoustic impedance $\hat{Z}_{\text {duct }}$ of the coupling duct

123 seen from the EC. This acoustic impedance is given by $\hat{Z}_{\text {duct }}=-j \hat{Z}_{e q}^{\text {duct }} \cot \hat{k}_{e q}^{\text {duct }} l_{\text {duct }} / \pi r_{\text {duct }}^{2}$ with the

124 duct length $l_{\text {duct }}$, its radius $r_{\text {duct }}$, the imaginary number $j$, and the equivalent wavenumber $\hat{k}_{e q}^{\text {duct }}$ and

125 characteristic impedance $\hat{Z}_{e q}^{\text {duct }}$ accounting for visco-thermal losses in the duct, calculated using a low 126 reduced frequency (LRF) model detailed in Appx. B. Since the duct inner cross-section is smaller than 127 the EC entrance area, a discontinuity occurs at the EC entrance and implies an inertia effect at the 128 medial aperture of the coupling duct. The acoustic mass of discontinuity is given by $129 L_{\text {dis }}=8 \rho_{0} H \alpha / 3 \pi^{2} r_{\text {duct }}$ [30]. $H \alpha$ corresponds to the correction factor of discontinuity as a 130 function of $\alpha$, the ratio between the duct and the equivalent EC entrance radius, and can be 131 approximated by $H \alpha=1-1.25 \alpha$ for large discontinuities [31] and to zero for negligible 132 discontinuities. 

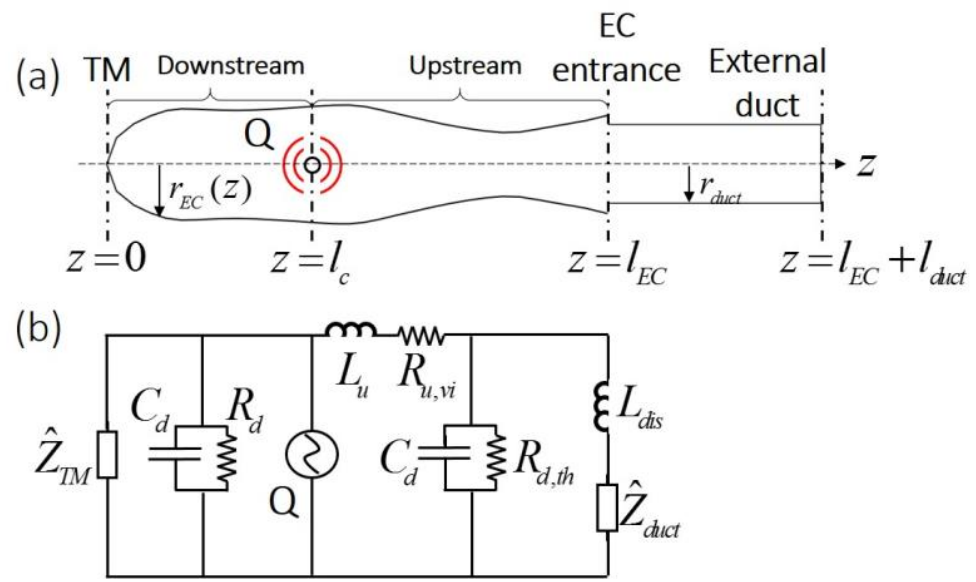

Figure 2: (a) Geometry of the EC coupled to the external capped duct and (b) its corresponding EA model. The tympanic membrane (TM) is indicated at $z=0$.

\subsection{Numerical investigation of the indirect method}

134 In this paper, the proposed indirect method is numerically investigated using an acousto-mechanical

135 3D FE model of the outer ear open and occluded by an external capped duct. Also, an associated purely

136 acoustical 3D FE model is presented. This model considerably decreases the computational time and is

137 therefore very practical to study the influence of several parameters of the coupled system which could

138 influence the accuracy of the method.

\subsubsection{Acousto-mechanical model}

140 The acousto-mechanical 3D FE model of an outer ear open and occluded by the external duct is

141 presented here. This outer ear model has been constructed by Brummund et al. [32] from cryosection

142 images of a female cadaver from the Visible Human Project ${ }^{\circledR}$. Recently, this model has been improved

143 by the authors [15]. The latter model is illustrated in Figure 3 (a) in the open case. The shape of the EC

144 is also displayed in Figure 4 assuming circular cross-sections. In the occluded case, the model is 145 adapted here to include the external duct (see Figure 3 (b)). The EC is surrounded by skin, cartilaginous 146 and bony tissues and a particular set of equivalent mechanical loading (reproducing a bone-conducted 147 stimulation) and boundary conditions is defined to reproduce a "plausible" vibration pattern of the EC 
148 wall [15]. The middle and inner ears are accounted for using the acoustic impedance $\hat{Z}_{T M}$ applied at the 149 tympanic membrane and defined by Shaw and Stinson's model [33] [34]. The vibration of the TM and 150 the ossicular chain induced by the bone-conducted stimulation are thus not accounted for. However, the 151 contribution of this vibration to the SPL generated in the EC is not expected to have significant 152 influence, at least at low frequencies below the middle ear resonance [16] [11] [10]. In the open case, 153 the acoustic impedance $\hat{Z}_{r a d}$ is defined at the EC entrance plane to account for the acoustic radiation in 154 the surrounding environment. In the occluded case, the coupling duct is made of aluminum whereas the 155 duct holder is made of silicone and fills a portion of the concha ahead of the EC entrance plane. The 156 external duct is coupled to the EC entrance in the normal direction and with a perfect seal. The outer 157 surface of the external duct portion which is not inserted in the duct holder is free. However, if the duct 158 is too long, it should be held in position at its lateral end. The influence of the boundary condition 159 (either free or fixed) defined at the lateral end circumference of the coupling duct (see Figure 3 (b)) is 160 studied in Sec. 3.1. 

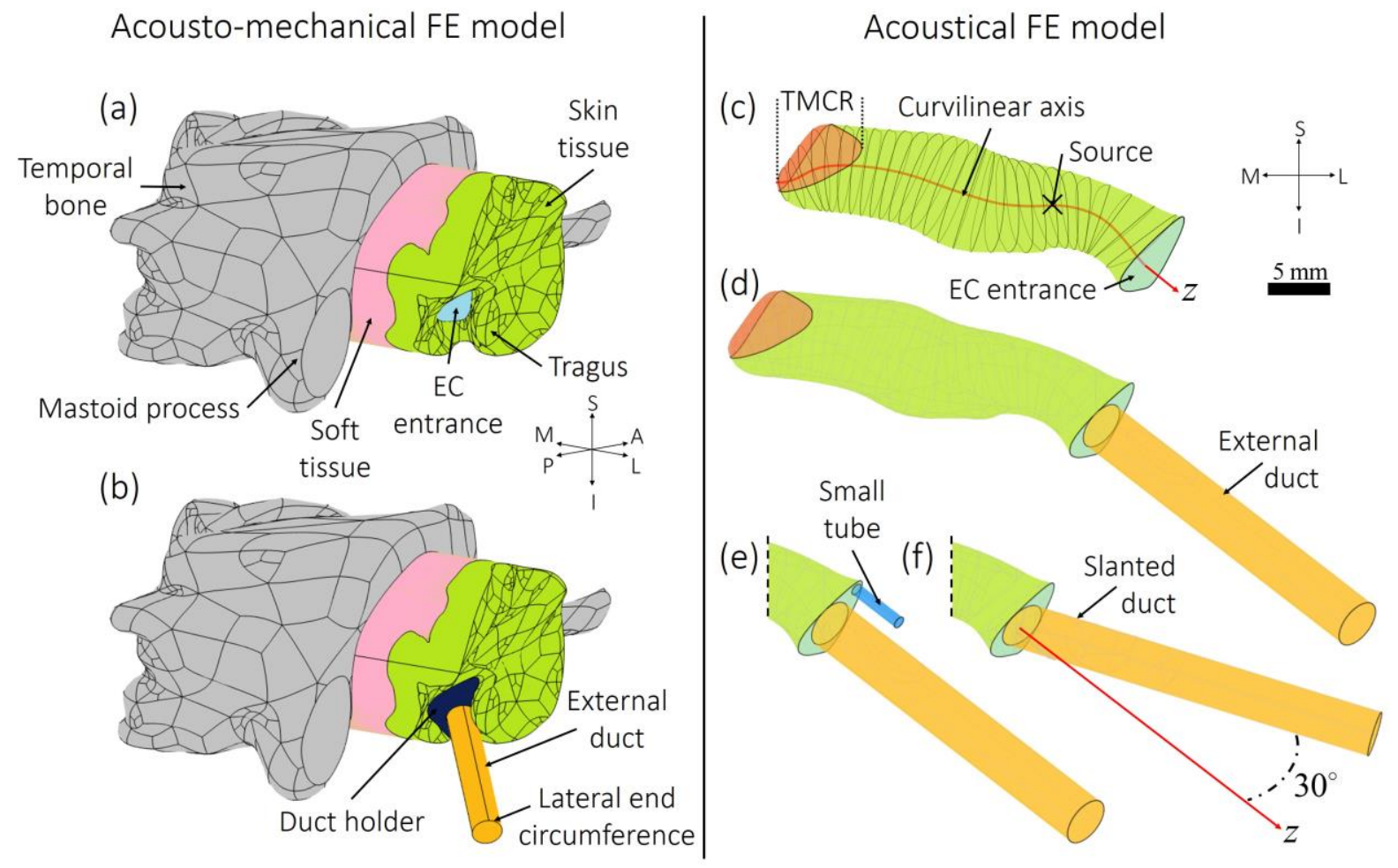

Figure 3: (Color online) Acousto-mechanical FE model of the outer ear (a) open and (b) occluded by an external capped duct coupled to the EC entrance using a duct holder; purely acoustical FE model of the EC (c) open and (d) occluded by the external duct normal to EC entrance plane. In subplot (e), an incomplete seal between the EC cavity and the duct is accounted for as a small tube coupled to the EC entrance. In subplot (f), the orientation of the duct forms an angle of $30^{\circ}$ with the normal to EC entrance plane. The coordinate system refers to superior $(\mathrm{S})$, inferior (I), posterior (P), anterior (A), medial (M) and lateral (L). 


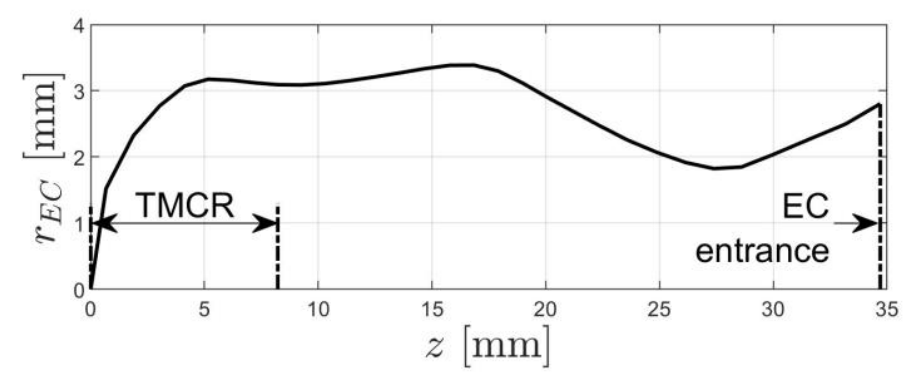

Figure 4: EC radius $r_{E C} z$ of the 3D FE model assuming circular cross-section and displayed as a function of the EC middle axis $z$. The tympanic membrane coupling region (TMCR) and the EC entrance are indicated.

161

162

163

164

165

166

167

168

169

170

171

172

173

174

175

\subsubsection{Purely acoustical model}

The purely acoustical 3D FE model of the EC open and occluded by the external capped duct is now presented (see Figure 3 (c) and (d)). The accuracy of the acoustical model is assessed against the acousto-mechanical model in Sec. 3.1. In the purely acoustical model, the EC and the coupling duct are rigidly walled (except the eardrum boundary where the acoustic impedance $\hat{Z}_{T M}$ is defined). The EC wall normal vibration is accounted for as an equivalent volume velocity source Q located on the EC middle axis at a curvilinear position $l_{c}^{F E}=23.6 \mathrm{~mm}$. This location corresponds to the curvilinear position of the EC wall normal velocity centroid computed using the acousto-mechanical 3D FE model [15].

In practice, an incomplete seal could occur at the EC entrance since it is a common issue in hearing protection for example. The geometry of an incomplete seal is generally unknown and is expected to be intricate [18]. It can be made of several interconnected pathways [35]. Here, this incomplete seal is modeled as a small tube of radius $r_{t}$ and length $l_{t}$ coupled to the EC entrance, in parallel with the external duct (see Figure 3 (e)). At the lateral end of the small tube, a radiation acoustic impedance of a circular baffled piston accounts for the coupling with the external environment. Furthermore, the 
external duct could be coupled in a direction that differs from the normal direction to the EC entrance

177 cross-section (see Figure 3 (f)). The influence of the incomplete seal and the duct orientation is

178 investigated in Secs. 3.2.5 and 3.2.3.

\section{2.3.3 Finite element modeling and computation of indicators}

180 In the acousto-mechanical 3D FE model, the sound propagation in solid domains is governed by the

181 linear elasto-dynamics equation relating the linearized displacement field $\underline{\hat{\hat{u}}}$, strain tensor $\underline{\underline{\hat{\xi}}}$, and stress

182 tensor $\stackrel{\hat{\sigma}}{=}$ at all points of solid domains [36]:

$$
\begin{gathered}
\underline{\nabla} \cdot \underline{\underline{\hat{\sigma}}}+\rho_{s} \omega^{2} \underline{\hat{u}}=0, \\
\underline{\hat{\sigma}}=\underline{\underline{\underline{E}}}: \underline{\underline{\hat{\xi}}}, \\
\underline{\underline{\hat{\xi}}}=\frac{1}{2} \underline{\nabla} \underline{\hat{u}}+\underline{\underline{v}}^{T},
\end{gathered}
$$

183 where $\rho_{s}$ is the solid density, $\omega$ is the angular frequency, $\underline{\nabla}$ is the nabla operator and $\underline{\underline{E}}$ is the fourth-

184 order stiffness (elasticity) tensor. In each solid domain, the structural damping is accounted for as a 185 multiplier $1+j \eta_{s}$ in the stiffness matrix where $\eta_{s}$ corresponds here to the isotropic loss factor.

186 Mechanical properties of skin, cartilaginous and bony tissues come from [15] and are summarized in

187 Table 8 (see Appx. A). In addition, Table 8 presents mechanical properties of the duct and the duct 188 holder.

189 In both acousto-mechanical and purely acoustical 3D FE models, the sound propagation in acoustic 190 domains is governed at all points by Helmholtz equation:

$$
\nabla^{2} \hat{p}+\hat{k}_{e q}^{2} \hat{p}=0,
$$


191 where $\hat{p}$ denotes the sound pressure and $\hat{k}_{e q}$ is the complex wavenumber accounting for viscous and 192 thermal losses using a LRF model (see Appx. B). Air properties used here are given in Table 9 (see 193 Appx. A).

194 At the interface between mechanical and acoustical domains, the continuity of stress is written as $195 \underline{\hat{\sigma}} \underline{n}=-\hat{p} \underline{n}$, where $\underline{n}$ is the normal vector at the interface, whereas the continuity of normal 196 displacements is given by $\frac{1}{\rho_{0} \omega^{2}} \partial \hat{p} / \partial n=\underline{\hat{u}} \cdot \underline{n}$.

197 In the acousto-mechanical FE model, a standard $(\underline{\hat{u}}, \hat{p})$ formulation is used to solve for both 198 displacements in solids and pressures in the fluid [36]. In the purely acoustical FE model, the problem 199 is only solved for pressures.

200 The geometry of each FE model is meshed according to a criterion of at least six 10-noded 201 (quadratic) tetrahedral elements per wavelength at $1 \mathrm{kHz}$ (maximum frequency of interest). The 202 wavelength, referred to as $\lambda$, is defined by $\lambda=c / f$ with $c$ the sound speed and $f$ the frequency. To 203 minimize the size of the mesh in solid structures of the acousto-mechanical FE model, the minimum 204 sound speed is taken between the compression and the shear wave speed defined respectively by

$$
c_{L}=\sqrt{\frac{E(1-\nu)}{\rho(1+\nu)(1-2 \nu)}},
$$

205 and

$$
c_{T}=\sqrt{\frac{G}{\rho}},
$$

206 with $E, \rho, \nu$ and $G$ the Young's modulus, density, Poisson's ratio and shear modulus respectively. 
Figure 5 shows the mesh of both the acousto-mechanical FE model and the purely acoustical FE 208 model using the mesh criteria previously detailed. It is noteworthy that the size of the mesh is also 209 constrained to capture the geometry of each domain. A convergence study has been performed on both 210 FE models to ensure the accuracy of simulation results presented in this paper. The current FE models 211 have been solved using COMSOL Multiphysics® (Sweden).

212 The surface averaged acoustic pressure computed at the tympanic membrane is referred to as $\left\langle\hat{p}_{T M}^{k}\right\rangle$, 213 where $k \in$ open, occl . Using the acousto-mechanical 3D FE model only, the centroid position vector 214 is computed by

$$
\underline{x}_{c}=\frac{\int_{S_{\text {wall }}} \underline{x}\left|\hat{v}_{n, \text { wall }} \quad \underline{x}\right| \mathrm{d} S}{\int_{S_{\text {wall }}}\left|\hat{v}_{n, \text { wall }} \quad \underline{x}\right| \mathrm{d} S},
$$

215 where $\underline{x}$ is the position vector in 3D space, $S_{\text {wall }}$ is the EC wall surface and $\hat{v}_{n, \text { wall }} \underline{x}$ is the complex 216 valued EC wall normal velocity. From Eq. (1), the curvilinear position $l_{c}^{F E}$ of the centroid position is 217 obtained by the normal projection of $\underline{x}_{c}$ on the EC middle axis and it is used in the purely acoustical 3D 218 FE model as mentioned previously. 


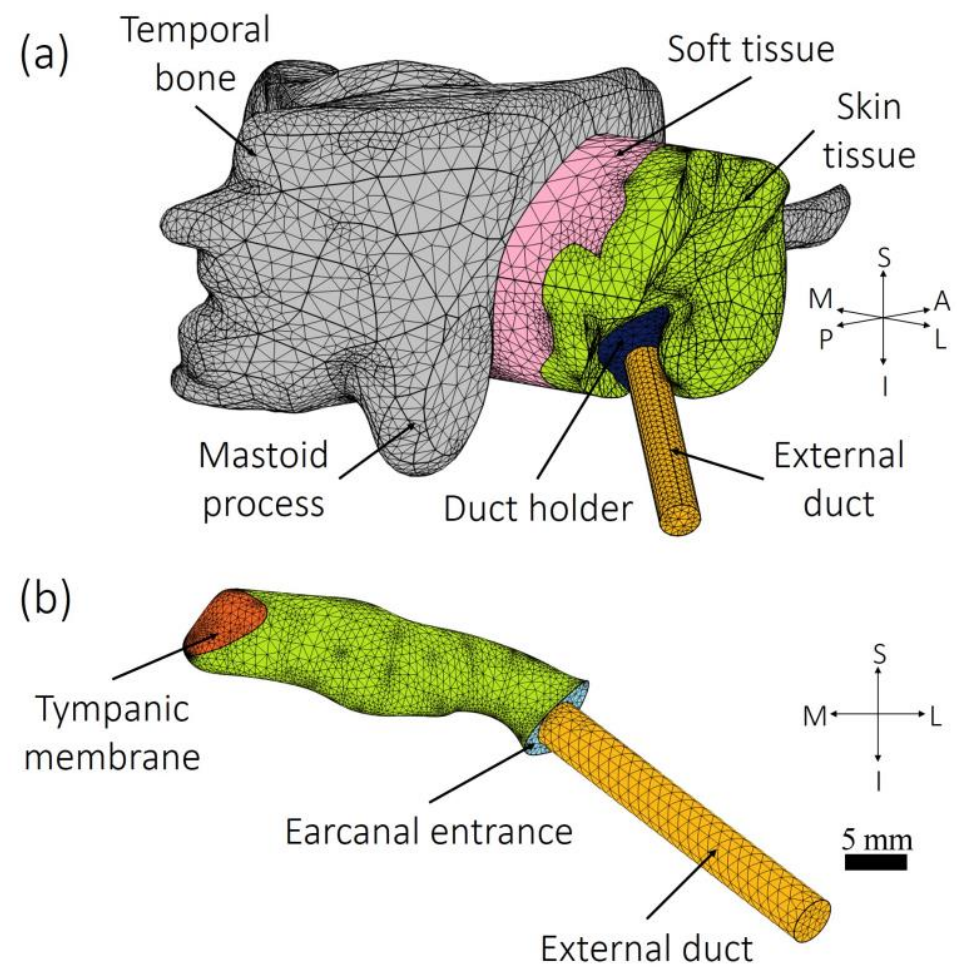

Figure 5: Mesh of (a) the acousto-mechanical and (b) purely acoustical 3D FE model of the outer ear occluded by the external duct. The coordinate system refers to superior $(\mathrm{S})$, inferior $(\mathrm{I})$, posterior $(\mathrm{P})$, anterior (A), medial (M) and lateral (L).

\section{Results and discussions}

This section is organized as follows. In Sec. 3.1, the centroid position of the EC wall normal velocity is estimated using the inverse method proposed in this paper. In Sec. 3.2, the influence of several parameters of the coupling system is numerically investigated. Finally, Sec. 3.3 briefly discusses the limitations of the work presented herein.

\subsection{Estimation of the centroid position from acoustic pressure transfer function}

In this section, the experimental setup (see Sec. 2.1) of the proposed method is simulated using both 3D FE models (see Sec. 2.3). The aim is to verify the accuracy of the purely acoustical FE model compared to the acousto-mechanical FE model taken as reference. Then, the purely acoustical FE 
model will be used in the parametric study (see Sec. 3.2) because of its low needs for computational resources. Then, the centroid position of the virtual outer ear of interest is estimated using the associated EA model (see Sec. 2.2) and compared to the centroid position directly computed using the acousto-mechanical 3D FE model in order to validate the indirect method.

Figure 6 (a) and (b) display respectively the level in $\mathrm{dB}$ and the phase of the tympanic membrane acoustic pressure transfer function between the EC open and occluded by an external capped duct computed as a function of frequency using the acousto-mechanical 3D FE model. Simulations considering both free (red squares) and fixed (blue stars) boundary conditions of the lateral end of the coupling duct are presented. The coupling duct has a length of $150 \mathrm{~mm}$, an inner radius of $2 \mathrm{~mm}$ and a wall thickness of $0.2 \mathrm{~mm}$ and is normally coupled to the EC entrance with a perfect seal. Note that the level in $\mathrm{dB}$ (factor 20) of the tympanic membrane acoustic pressure transfer function corresponds to the objective OE. According to Figure 6 (a), the transfer function amplitude reaches a local minimum and a local maximum. Figure 6 also displays the results computed using the purely acoustical 3D FE model in which the EC wall normal vibration is modeled as an equivalent ideal source of volume velocity located at the centroid position of the corresponding vibration (see black line). This model gives the same results as the acousto-mechanical FE model (including both boundary conditions of the coupling duct), at least up to the transfer function local minimum frequency. Above this frequency, slight discrepancies are observed between both models due to the elastic modes of deformation of the duct holder and the coupling duct itself (not accounted for in the purely acoustical model). In the acoustomechanical FE model, the boundary condition defined at the lateral end of the coupling duct (either free or fixed) is shown to have little influence on the results displayed in Figure 6. According to Figure 6 (a) and (b), local extrema of the transfer function amplitude are associated with sudden phase shifts which characterize the occurrence of acoustic antiresonance (local minimum at $516 \mathrm{~Hz}$ ) and acoustic resonance (local maximum at $725 \mathrm{~Hz}$ ) in the EC coupled to the duct. 
To illustrate these phenomena, Figure 7 displays the acoustic pressure field (in $\mathrm{dB}$ ) in the coupled system in (a) antiresonance and (b) resonance states computed using the purely 3D FE model. In Figure

7 (a), the acoustic pressure level is minimum in the region between the eardrum and the centroid

position of the EC wall normal velocity and gradually increases to reach a maximum value at the

capped lateral end of the duct. A quarter-wavelength acoustic resonance occurs in the EC upstream

section (from the centroid position to the EC entrance) coupled to the external duct and corresponds to

an antiresonance seen by the EC wall normal vibration which encounters a pronounced acoustic

pressure dip at its centroid position. The acoustic pressure is also approximately minimum up to the

eardrum because the vibro-acoustic behavior of the EC downstream section (from the centroid position

to the eardrum) is governed by its compressibility effect [15]. In Figure 7 (b), the acoustic pressure

level is maximum at both the eardrum and the capped lateral end of the coupling duct and minimum in

between. This standing wave pattern is characteristic of a half-wavelength acoustic resonance of the

whole coupled system excited by the EC wall normal vibration. Unlike resonance, antiresonance

depends on the location of the source which corresponds to the centroid position of the EC wall normal vibration. Therefore, acoustic antiresonance can be used to estimate the centroid position.

To the authors' knowledge, no experimental data of the transfer function displayed in Figure 6 exist in the literature. In 1960, however, Huizing [8] measured the objective OE induced by an external capped duct on a human subject for several duct lengths for a bone-conducted stimulation at a frequency $1 \mathrm{kHz}$. Huizing conducted this experiment to highlight a standing wave pattern in the EC. In Appx. C, Huizing's experiment is simulated using the purely acoustical 3D FE model and both simulated and measured objective $\mathrm{OE}$ are shown to be in relatively good agreement. Huizing interpreted OE minima as acoustic resonances with an acoustic pressure node at the eardrum. Several 
275 interpreted in the same way, assuming a low enough acoustic impedance of the tympanic membrane. 276 However, as previously explained, OE minima rather come from acoustic antiresonances with an 277 acoustic node at the centroid position of the EC wall normal velocity (see Figure 7 (a)). At 278 antiresonances, the acoustic pressure is also minimum at the eardrum for the reason explained above 279 (i.e., EC downstream section governed by its compressibility effect).

280 The antiresonance frequency of the coupled system is then determined from the transfer function of 281 Figure 6 and corresponds to the amplitude minimum which is also associated with the sudden phase 282 shift. For the $150 \mathrm{~mm}$ duct considered here, the antiresonance frequency is equal to $516 \mathrm{~Hz}$. It is 283 noteworthy that the antiresonance frequency could be determined directly from the acoustic pressure 284 measured in the occluded EC. The use of the OE, however, has the advantage to avoid the influence of 285 variations of the EC wall normal vibration (amplitude and phase) with frequency on the antiresonance 286 frequency determination (not shown here).

From the antiresonance frequency determined using the 3D FE models (both models provide the same antiresonance frequency), the centroid position is estimated using the EA model. For this 289 purpose, the antiresonance frequency of the coupled system is computed using the EA model as a function of the source position included between the tympanic membrane coupling region and the EC entrance. Then, the centroid position is obtained by minimizing the difference between the 292 antiresonance frequency determined from the 3D FE model and that computed by the EA model as a function of the source position. In the configuration of interest, the EA model estimates the centroid position of the EC wall normal vibration with a slight difference of $0.3 \mathrm{~mm}$ compared to the $3 \mathrm{D} \mathrm{FE}$ model. This difference does not come from a lack of convergence of the method (this has been checked but not shown here for conciseness), but is more likely due to the planar waves assumption used by the 
297 EA model. This assumption could be debatable mainly in the coupling region between the EC and the 298 external duct and to a lesser extent in the tympanic membrane coupling region.

299 Regarding in particular the coupling region between the EC and the duct, the cross-sectional 300 discontinuity is accounted for, in the EA model, as an inertia effect of acoustic mass $L_{d i s}$ added to the 301 input acoustic impedance $\hat{Z}_{d u c t}$ of the duct (see Sec. 2.2). The acoustic mass is calculated from Karal's 302 formula [30] developed for the coupling between two cylindrical ducts of constant radius. Here, 303 however, the cross-section of the EC is not circular and its shape varies along the EC middle axis. 304 Schmidt and Hudde [38] underlined that the mass of discontinuity is critically dependent on the shape 305 of the EC entrance region. Therefore, the EA model cannot exactly account for the influence of the EC 306 entrance discontinuity and, in consequence, it cannot provide an exact estimation of the centroid 307 position. A difference lower than $0.5 \mathrm{~mm}$ is however considered acceptable.

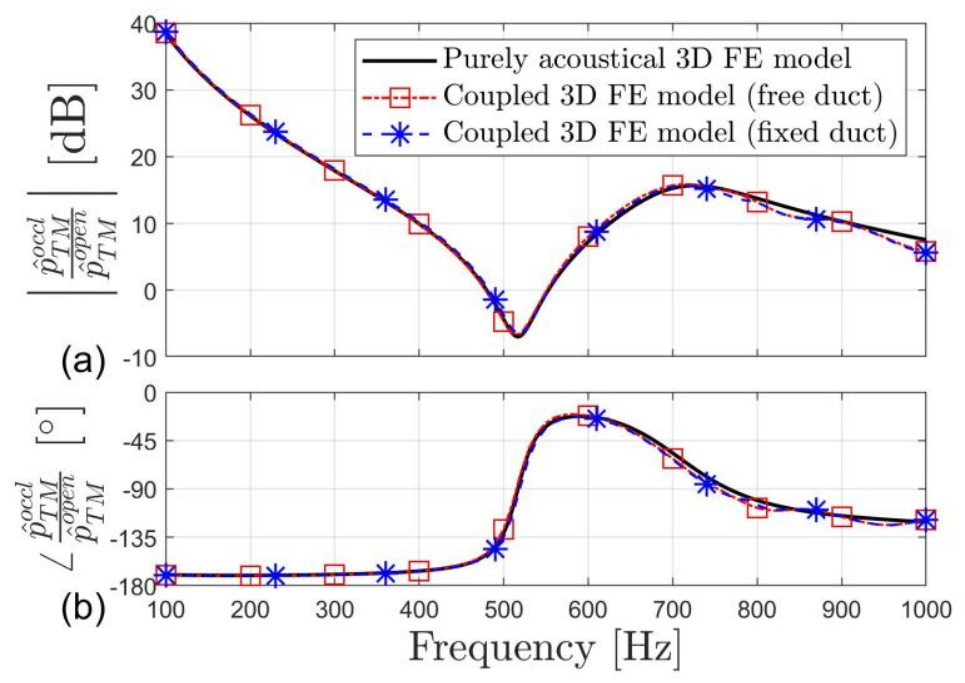

Figure 6: (a) Level in $\mathrm{dB}\left(20 \log _{10}\right.$, ref. 1) and (b) phase of the tympanic membrane acoustic pressure ratio between the occluded and the open EC computed using the purely acoustical and the acoustomechanical 3D FE models. The occlusion is ensured by a capped duct coupled at the EC entrance of $150 \mathrm{~mm}$ length, $2 \mathrm{~mm}$ inner radius, and, in the acousto-mechanical model only, $0.2 \mathrm{~mm}$ wall thickness. In the acousto-mechanical model, the boundary condition at the external end of the duct is free or fixed. 


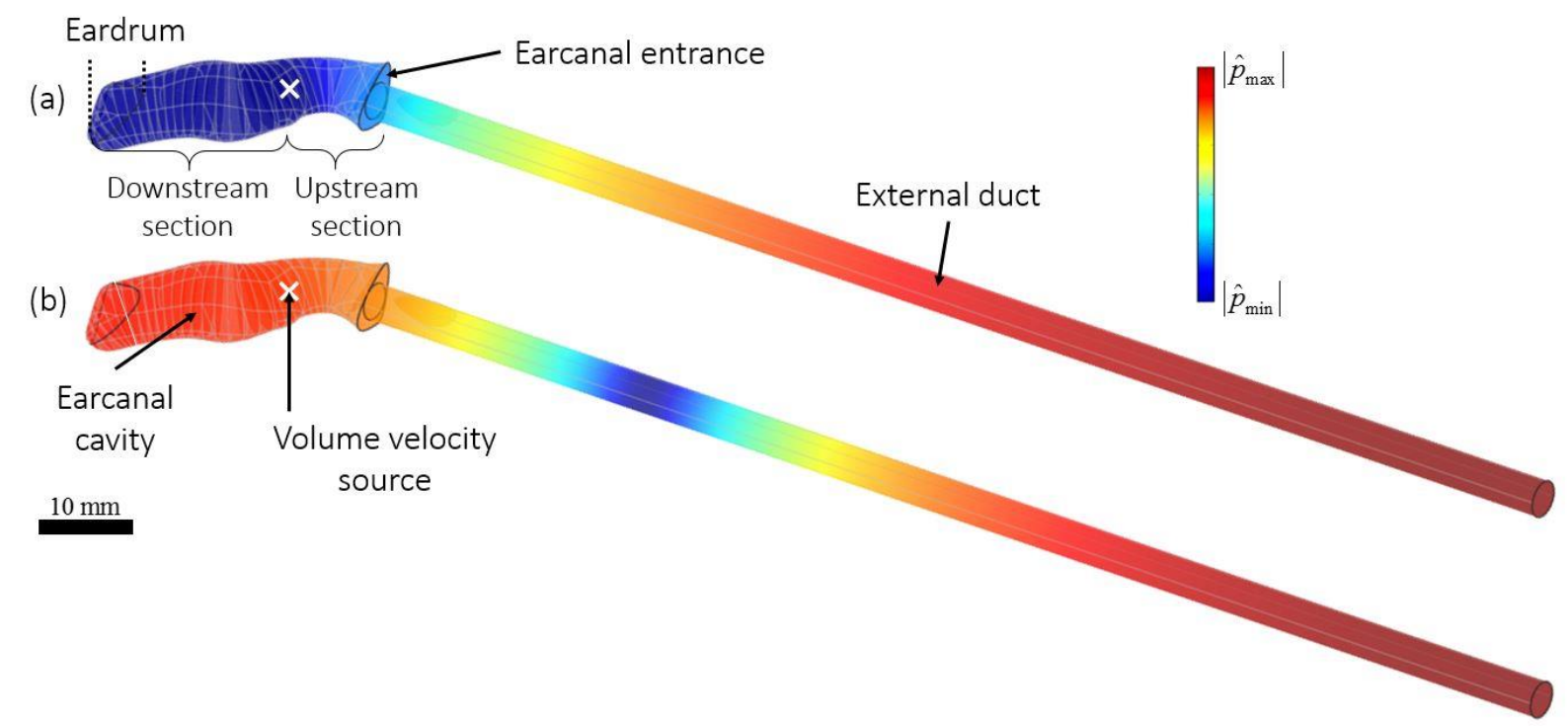

Figure 7: (Color online) Acoustic pressure field in $\mathrm{dB}\left(20 \log _{10}\right.$, ref. $\left.2 \times 10^{-5} \mathrm{~Pa}\right)$ in the EC coupled to an external capped duct of length $150 \mathrm{~mm}$ and inner radius $2 \mathrm{~mm}$ in (a) antiresonance (516 Hz) and (b) resonance $(725 \mathrm{~Hz})$ states computed using the acoustical 3D FE model.

\subsection{Influence of several parameters of the coupled system}

This section investigates the influence of several parameters of the coupled system on the estimation of the centroid position using the proposed method. These parameters are the length of the duct, its

311 radius, the duct orientation, the coupling position of the duct in the EC, the presence of an incomplete 312 seal between the coupling duct and the EC entrance, the EC shape, the temperature in the coupled system and the EC downstream section and tympanic membrane acoustic impedance. In this section, 314 the purely acoustical FE model is used instead of the acousto-mechanical FE model since it requires 315 significantly less computational resources.

\subsubsection{Length of the coupling duct and sensitivity of the indirect method}

In the acousto-mechanical 3D FE model, the centroid position of the EC wall normal velocity does not significantly vary between $100 \mathrm{~Hz}$ and $1 \mathrm{kHz}$ [15]. In practice, however, it could be possible that the centroid position varies in the low frequency range of interest. Since the proposed method estimates 
be used to cover the frequency range $(100 \mathrm{~Hz}$ to $1 \mathrm{kHz})$. Here, in addition to the $150 \mathrm{~mm}$ duct used in 322 Sec. 3.1, two additional duct lengths are used: $750 \mathrm{~mm}$ and $75 \mathrm{~mm}$. In the case of a $750 \mathrm{~mm}$ duct, 323 several antiresonances occur between $100 \mathrm{~Hz}$ and $1 \mathrm{kHz}$ but only the lowest frequency is considered 324 here. Table 1 presents the first antiresonance frequency $f_{\text {antires }}^{F E}$ of the EC coupled to these three external 325 ducts and computed using the acoustical 3D FE model. The longer the coupling duct, the lower the first 326 antiresonance frequency of the coupled system. The EA model is now used to estimate the centroid 327 position from the antiresonance frequencies $f_{\text {antires }}^{F E}$. Table 1 presents the difference $\Delta l_{c}$ between the 328 centroid position $l_{c}^{E A}$ estimated by the EA model and the effective centroid position $l_{c}^{F E}$ used in the 329 acoustical 3D FE model. According to Table 1, this difference is maximum for the longest duct $330(+1.2 \mathrm{~mm})$ and minimum for the shortest $(-0.1 \mathrm{~mm})$. As discussed in Sec. 3.1, the error of estimation of 331 the EA model mainly comes from the way the discontinuity at the EC entrance is accounted for 332 between the EC and the coupling duct. The reason why this error increases with the length of the 333 coupling duct is however related to the sensitivity of the method which is now investigated depending 334 on the duct length.

Table 1: Antiresonance frequency of the coupled system depending on the external duct length computed using the 3D FE models. The centroid position estimated using the EA model is also presented in each case (relative to the effective position computed using the 3D FE model).

\begin{tabular}{|c|c|c|}
\hline$l_{\text {duct }}[\mathrm{mm}]$ & $\begin{array}{c}f_{\text {antires }}^{F E}[\mathrm{~Hz}], \\
\text { FE model }\end{array}$ & $\begin{array}{c}\Delta l_{c}=l_{c}^{E A}-l_{c}^{F E} \\
{[\mathrm{~mm}]}\end{array}$ \\
\hline 750 & 104 & +1.2 \\
\hline 150 & 516 & +0.3 \\
\hline 75 & 980 & -0.1 \\
\hline
\end{tabular}

In order to illustrate the calculation of the method sensitivity, Figure 8 displays the antiresonance 336 frequency of the coupled system as a function of the source position $l_{c}$ computed using the EA model 337 for the three duct lengths $(750,150$ and $75 \mathrm{~mm})$. In practice, the centroid position is not expected to lie 
338 in the tympanic membrane coupling region (TMCR) so this region is excluded from the variation range 339 of the source. For each duct, the antiresonance frequency increases with $l_{c}$ because the distance 340 between the source and the capped lateral end of the duct decreases. More importantly, Figure 8 shows 341 that the range of variation of the antiresonance frequency is minimum for the longest duct and 342 maximum for the shortest duct. The antiresonance frequency of the coupled system is more sensitive to 343 a variation of the centroid position when the coupling duct is short and vice versa. The mean sensitivity 344 of the method, referred to as $\bar{\sigma}$ and expressed in $\mathrm{Hz} \mathrm{mm}^{-1}$, can be computed as the ratio between the 345 antiresonance frequency interval and the source position interval. For the $750 \mathrm{~mm}$ duct, $346 \bar{\sigma}=105-103 / 34.7-8 \approx 0.1 \mathrm{~Hz} \mathrm{~mm}^{-1}$. For the $150 \mathrm{~mm}$, the mean sensitivity of the method is 347 equal to approximately $2 \mathrm{~Hz} \mathrm{~mm}^{-1}$ and to about $8 \mathrm{~Hz} \mathrm{~mm}^{-1}$ for the $75 \mathrm{~mm}$ duct. It is not yet certain with 348 which precision the centroid position should be estimated. It surely depends on the use of this position 349 (e.g., input of an EA model, validation of a FE model, study of the influence of a bone-conducted 350 source to the EC wall vibration distribution). It is certain, however, that the antiresonance frequency 351 cannot be identified from experimental data with an accuracy of $0.1 \mathrm{~Hz}$. In fact, it would be probably 352 tricky to obtain the antiresonance frequency with an accuracy of $1 \mathrm{~Hz}$. In this condition, it could not be 353 possible to accurately estimate the centroid position with the proposed method below approximately $354500 \mathrm{~Hz}$ (i.e., for a coupling duct longer than $150 \mathrm{~mm}$ ). In the following, the $750 \mathrm{~mm}$ duct is therefore 355 not considered anymore. 


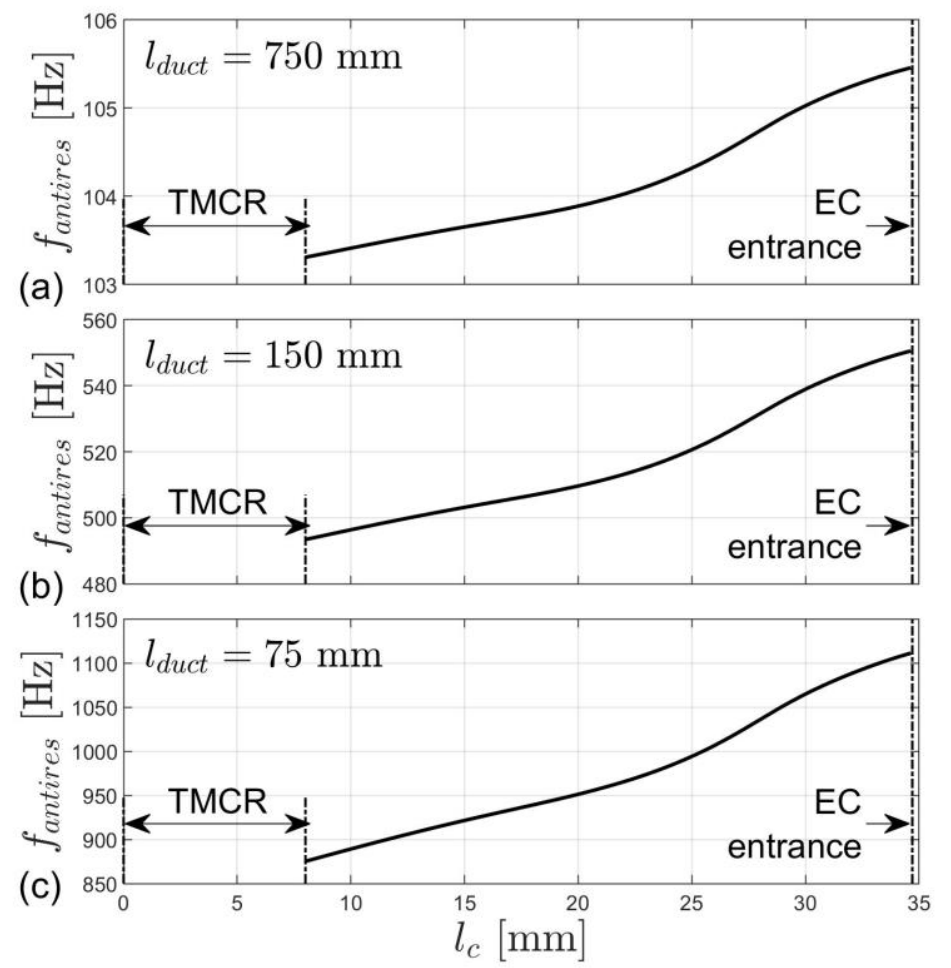

Figure 8: Antiresonance frequency computed as a function of the source position comprised between the tympanic membrane coupling region (TMCR) and the EC entrance using the EA model for three duct lengths. In each case, the duct inner radius is equal to $2 \mathrm{~mm}$.

\subsubsection{Inner radius of the coupling duct}

357 In previous simulations, the inner radius of the duct has been chosen equal to $2 \mathrm{~mm}$ and corresponds 358 to the largest admissible duct radius for the EC entrance considered here. In this section, the influence 359 of the duct radius on the mean sensitivity of the method is investigated. Figure 9 displays the mean 360 sensitivity of the method as a function of the duct radius computed using the EA model for two lengths 361 of the coupling duct $(150 \mathrm{~mm}$ and $75 \mathrm{~mm})$. The duct radius $r_{d u c t}$ decreases from the equivalent radius $362 r_{E C} l_{E C}=2.8 \mathrm{~mm}$ of the EC entrance (assuming circular cross-section) to almost $0.5 \mathrm{~mm}$. For each 363 duct radius, the mean sensitivity of the method is higher for the $75 \mathrm{~mm}$ duct compared to the $150 \mathrm{~mm}$ 364 duct for the reason explained in Sec. 3.2.1. For both ducts, the mean sensitivity of the method would be 365 maximum if the duct could have the same radius as the EC entrance (assuming circular cross-section). 
366 The mean sensitivity tends to zero when the duct radius diminishes. Indeed, when the radius of the 367 coupling duct decreases, the modulus of its acoustic impedance $\hat{Z}_{d u c t}$ seen from the EC entrance 368 increases, mainly due to (i) the sudden change in section between the EC entrance and the duct and (ii) 369 the augmentation of visco-thermal losses in the coupling duct (see Sec. 2.2). In consequence, the EC 370 upstream section gradually shifts from a mass-controlled vibro-acoustic behavior governed by the 371 acoustic mass $L_{u}$ to a compliance-controlled vibro-acoustic behavior dominated by the acoustic 372 compliance $C_{u}$ (see Figure 2 (b)). The antiresonance frequency of the coupled system thus tends to the 373 quarter-wavelength resonance of the external duct and becomes independent from the centroid position. 374 Since the influence of the centroid position on the antiresonance frequency of the coupled system 375 vanishes when the duct radius decreases, the mean sensitivity of the method tends to zero (see Figure 376 9). For small enough duct radius (below approximately $0.5 \mathrm{~mm}$ ), the antiresonance phenomenon itself 377 vanishes in the low frequency range of interest because there is no more wave propagation in the 378 coupling duct due to visco-thermal damping and the EC coupled to the duct behaves as if the EC were 379 occluded at its entrance (no coupling duct). To perform the method and maximize its accuracy, the 380 outer radius of the coupling duct must be chosen as large as possible (in the limit, however, of the size 381 of the EC entrance) whereas the thickness of its wall should be minimum.

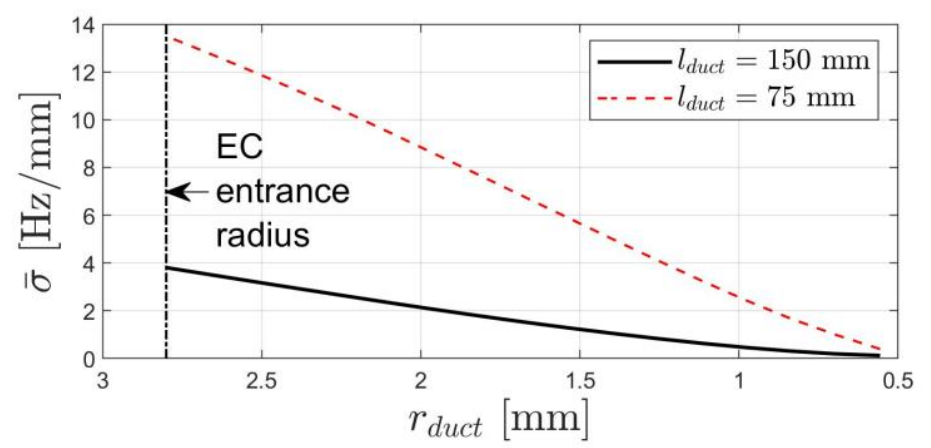

Figure 9: Mean sensitivity of the indirect method computed using the EA model as a function of the duct radius for two lengths of the external duct (150 $\mathrm{mm}$ and $75 \mathrm{~mm})$. 
In previous simulations, the direction of the coupling duct has been assumed normal to the plane of

384 the EC entrance. In practice, however, when the contemplated physical measurements are made, the 385 direction of the duct is constrained by the pinna and the tragus. Therefore, it would be certainly 386 difficult, if not impossible, to ensure the coupling in the exact normal direction of the EC entrance 387 plane. In this section, the influence of a variation of the duct orientation on the accuracy of the method 388 is studied for the two duct lengths 150 and $75 \mathrm{~mm}$. For this purpose, the duct orientation is changed by $38930^{\circ}$ from the EC entrance normal direction (see Figure 3 (f)). Similarly to what proposed Schmidt and 390 Hudde [38], the rotation of the coupling duct is performed around a fixed point corresponding to the 391 intersection of the EC middle axis with the EC entrance plane where the duct is coupled. Therefore, the 392 curvilinear length of the EC remains untouched, as well as the length of the coupling duct. The change 393 in orientation, however, increases the area of the coupling between the EC entrance and the duct. Table 3942 presents the corresponding change in antiresonance frequency computed using the 3D FE model. The 395 coupling angle slightly increases the antiresonance frequency of the coupled system. Indeed, due to the 396 increase of the coupling area, the associated acoustic mass of discontinuity decreases and therefore the 397 apparent length of the coupled system also diminishes. Table 2 also presents the variation $\Delta l_{c}$ of the 398 centroid position induced by the change in orientation of the duct and estimated using the EA model 399 from antiresonance frequencies predicted by the FE model. According to Table $2, \Delta l_{c}$ is close to $400 \quad 0.3 \mathrm{~mm}$ for each duct. The method proposed here to estimate the centroid position of the EC wall 401 normal velocity is thus little influenced by the duct orientation in the range of variation considered 402 here. 
Table 2: Influence of the duct orientation on the antiresonance frequency computed using the 3D FE model. The influence on the centroid position estimated using the EA model is also presented.

\begin{tabular}{|c|c|c|c|}
\hline$l_{\text {duct }}[\mathrm{mm}]$ & $\begin{array}{c}f_{\text {antires }}^{F E}[\mathrm{~Hz}], \\
\text { normal direction }\end{array}$ & $\begin{array}{c}f_{\text {antires }}^{F E}[\mathrm{~Hz}], \\
30^{\circ} \text { slanted }\end{array}$ & $\Delta l_{c}[\mathrm{~mm}]$ \\
\hline 150 & 516 & 516.5 & +0.3 \\
\hline 75 & 980 & 982.4 & +0.3 \\
\hline
\end{tabular}

\subsubsection{Coupling position of the external duct}

In previous simulations, the external capped duct has been exactly coupled to the EC entrance plane. In practice, however, the coupling position of the duct could be slightly more inside or outside the EC compared to the chosen position of the EC entrance plane used in the EA model involved in the indirect method. This section thus investigates the influence of this uncertainty on the estimated centroid position. Table 3 presents the change in antiresonance frequency of the coupled system computed using the EA model due to a shift in the coupling position from $+3 \mathrm{~mm}$ (outward the EC entrance plane) to $3 \mathrm{~mm}$ (inward the EC entrance plane). Two lengths of coupling duct are considered (150 and $75 \mathrm{~mm}$ ). In each case, the inner radius of the duct is equal to $2 \mathrm{~mm}$. For both ducts, the antiresonance frequency is the highest when the coupling position is the farthest inside the EC (see Table 3). This is explained by the reduction of the distance between the centroid position and the lateral capped end of the duct. Table 3 also presents the variation $\Delta l_{c}$ of the estimated centroid position due to an error in the coupling position. The estimation is performed using the EA model assuming that the external duct is exactly coupled to the EC entrance plane (i.e., a coupling position variation of $0 \mathrm{~mm}$ ). When the effective coupling position is shifted outside the EC, the centroid position is estimated further in the EC, and vice versa (see Table 3). Also, the error in the estimated centroid position is lower than the coupling position shift (in absolute value). This error, however, depends on the EC shape and tends to be equal to the coupling position shift when the EC shape is straight (not shown in Table 3). The maximum error in the estimated centroid position is thus bounded by the coupling position shift. 
Table 3: Influence of the coupling position (relative to the EC entrance position) on the antiresonance frequency computed using the EA model. The influence on the centroid position estimated using the EA model is also presented.

\begin{tabular}{|c|c|c|c|}
\hline$l_{\text {duct }}[\mathrm{mm}]$ & $\begin{array}{c}\text { Coupling } \\
\text { position }[\mathrm{mm}]\end{array}$ & $f_{\text {antires }}^{E A}[\mathrm{~Hz}]$ & $\Delta l_{c}[\mathrm{~mm}]$ \\
\hline \multirow{3}{*}{150} & +3 & 512 & -1.9 \\
\cline { 2 - 4 } & +1.5 & 514 & -0.8 \\
\cline { 2 - 4 } & 0 & 517 & 0 \\
\cline { 2 - 4 } & -1.5 & 520 & +0.9 \\
\cline { 2 - 4 } & -3 & 523 & +2.3 \\
\hline 75 & +3 & 961 & -2.2 \\
\cline { 2 - 4 } & +1.5 & 970 & -1.2 \\
\cline { 2 - 4 } & 0 & 979 & 0 \\
\cline { 2 - 4 } & -1.5 & 990 & +0.9 \\
\cline { 2 - 4 } & -3 & 1005 & +2.3 \\
\hline
\end{tabular}

422

\subsubsection{Incomplete seal at the earcanal/duct junction}

In previous simulations, a perfect seal has been assumed between the coupling duct and the EC wall at the EC entrance. In this section, the influence of an incomplete seal on the accuracy of the method is investigated using the 3D FE model for two duct lengths $150 \mathrm{~mm}$ and $75 \mathrm{~mm}$. The incomplete seal is modeled as a small cylindrical tube of radius $r_{t}$ and length $l_{t}$ coupled at the EC entrance in parallel with the external coupling duct (see Figure $3(\mathrm{e}))$. Two "plausible" values of radius $(0.4$ and $0.6 \mathrm{~mm})$ and length $(5$ and $10 \mathrm{~mm})$ are chosen to illustrate the influence of an incomplete seal. Table 4 presents the change in antiresonance frequency computed using the 3D FE model. Table 4 also presents the variation $\Delta l_{c}$ of the centroid position induced by incomplete seals and estimated using the EA model from antiresonance frequencies predicted by the FE model. According to Table 4, the influence of the incomplete seal on the estimated centroid position is similar for each coupling duct. In addition, this influence decreases when the length of the incomplete seal increases and when its radius decreases. This phenomenon is due to the augmentation of both the acoustic mass and the acoustic resistance of 
435 the incomplete seal which increases its acoustic impedance and thus decreases the volume velocity 436 transfer through it. The influence of an incomplete seal on the acoustic pressure field of the coupled 437 system is very small around the antiresonance frequency because the acoustic pressure is already 438 minimum in the EC (see Figure 7 (a)). The influence of an incomplete seal is rather of significant 439 importance when the acoustic pressure is maximum in the EC (e.g., at low frequencies for an occlusion 440 ensured at the EC entrance [18]). However, in order to maximize the accuracy of the proposed method, 441 an incomplete seal must be avoided as much as possible. The quality of the seal could be assessed 442 using fit-testing method developed for hearing protection [39] [40].

Table 4: Influence of an incomplete seal between the duct and the EC entrance on the antiresonance frequency computed using the 3D FE model. The incomplete seal is modeled as a small tube of radius $r_{t}$ and length $l_{t}$ connected to the EC entrance in parallel with the coupling duct. The influence on the centroid position estimated using the EA model is also presented.

\begin{tabular}{|c|c|c|c|c|}
\hline$l_{\text {duct }}[\mathrm{mm}]$ & $\begin{array}{c}f_{\text {antires }}^{F E}[\mathrm{~Hz}], \\
\text { perfect seal }\end{array}$ & \multicolumn{2}{|c|}{$\begin{array}{c}f_{\text {antires }}^{F E}[\mathrm{~Hz}], \\
\text { incomplete seal }\end{array}$} & $\Delta l_{c}[\mathrm{~mm}]$ \\
\hline \multirow{2}{*}{150} & 516 & $r_{t}=0.4 \mathrm{~mm}, l_{t}=5 \mathrm{~mm}$ & 518 & +0.9 \\
\cline { 3 - 5 } & & $r_{t}=0.4 \mathrm{~mm}, l_{t}=10 \mathrm{~mm}$ & 517 & +0.5 \\
\cline { 3 - 5 } & & $r_{t}=0.6 \mathrm{~mm}, l_{t}=5 \mathrm{~mm}$ & 520 & +1.8 \\
\hline 75 & \multirow{2}{*}{980} & $r_{t}=0.4 \mathrm{~mm}, l_{t}=5 \mathrm{~mm}$ & 986 & +0.7 \\
\cline { 3 - 5 } & & $r_{t}=0.4 \mathrm{~mm}, l_{t}=10 \mathrm{~mm}$ & 983 & +0.4 \\
\cline { 3 - 5 } & & $r_{t}=0.6 \mathrm{~mm}, l_{t}=5 \mathrm{~mm}$ & 992 & +1.4 \\
\hline
\end{tabular}

\subsubsection{Straight cylindrical earcanal versus 3D earcanal shape}

444 In previous simulations, the shape of the EC was perfectly known. If the 3D shape could not be 445 measured, could the EC be assumed to a straight cylinder? This section investigates the error of 446 estimation of the centroid position associated with this assumption. For this purpose, a straight EC of 447 constant radius $2.8 \mathrm{~mm}$ and length $34.7 \mathrm{~mm}$ is used. The radius corresponds to the equivalent radius of 
448 the 3D EC entrance (assuming circular cross-section) whereas the length is equal to the curvilinear 449 length of the 3D EC. Table 5 presents the antiresonance frequency of the coupled system using the 3D 450 EC shape and the straight EC for two duct lengths (150 and $75 \mathrm{~mm})$. For both coupling ducts, higher 451 antiresonance frequencies are obtained with the straight EC compared to the real 3D EC shape. Due to 452 the absence of constriction close to the EC entrance in the straight EC compared to the 3D EC shape 453 (see Figure 4), the acoustic mass of the EC upstream section is smaller in the former than the latter EC. 454 In consequence, the effective length of the EC upstream section appears smaller in the straight EC than 455 in the 3D EC shape, explaining the antiresonance frequencies difference between both.

456 Table 5 also presents the influence of the EC shape on the estimated centroid position. The 457 estimation is performed from antiresonance frequencies of the 3D EC shape coupled to the external 458 ducts using the EA model which assumes a straight EC. According to Table 5, neglecting the 3D EC 459 shape induces significant errors in the estimation of the centroid position (around $8 \mathrm{~mm}$ towards the 460 eardrum) and, therefore, cannot be neglected. The magnitude of this error, however, depends on the 461 individual EC shape and how the latter differs from a cylindrical shape.

Table 5: Influence of the EC shape on the antiresonance frequency computed using the EA model. The influence on the centroid position estimated using the EA model is also presented.

\begin{tabular}{|c|c|c|c|}
\hline$l_{\text {duct }}[\mathrm{mm}]$ & $\begin{array}{c}f_{\text {antires }}^{E A}[\mathrm{~Hz}], \\
\text { 3D EC shape }\end{array}$ & $\begin{array}{c}f_{\text {antires }}^{E A}[\mathrm{~Hz}], \\
\text { Straight EC }\end{array}$ & $\Delta l_{c}[\mathrm{~mm}]$ \\
\hline 150 & 517 & 543 & -8.5 \\
\hline 75 & 979 & 1083 & -7.4 \\
\hline
\end{tabular}

\section{3.2.7 Temperature in the coupled system}

463 Air properties used in previous simulations approximately correspond to ambient temperature $19^{\circ} \mathrm{C}$, 464 atmospheric pressure $1013 \mathrm{hPa}$ and relative humidity $20 \%$. Appendix. D details the calculation of air 465 properties from temperature, atmospheric pressure and relative humidity. These values can be easily 
measured during the experiment. An uncertainty exists, however, regarding the temperature in the EC 467 occluded by the coupling duct. Indeed, assuming that the temperature of the $\mathrm{EC}$ wall is close to $37^{\circ} \mathrm{C}$ 468 while the ambient temperature is equal to $19^{\circ} \mathrm{C}$, a gradient of temperature occurs between the EC and 469 the coupling duct. Also, this gradient could change during the measurement, depending on the 470 measurement duration, due to heat transfer by convection.

471 In this section, the effect of the temperature is investigated. For this purpose, two additional 472 configurations are proposed: (i) the temperature in the $\mathrm{EC}$ is equal to $T_{E C}=37{ }^{\circ} \mathrm{C}$ whereas the 473 temperature in the coupling duct is equal to $T_{d u c t}=19^{\circ} \mathrm{C}$ and (ii) the temperature in the coupled system 474 is homogenous and equal to $37^{\circ} \mathrm{C}$. Table 6 presents the change in antiresonance frequency of the 475 coupled system computed using the EA model due to the variation in temperature. The augmentation of 476 the temperature in the EC only or in the entire coupled system increases the antiresonance frequency. 477 This is mainly explained by the increase of sound speed with temperature from 343 to $354 \mathrm{~m} \mathrm{~s}^{-1}$. Table 4786 also presents the variation $\Delta l_{c}$ of the estimated centroid position induced by the change in 479 antiresonance frequency due to the temperature. The estimation is performed using the EA model and 480 assuming a homogeneous temperature of $19^{\circ} \mathrm{C}$ in the entire coupled system as reference. According to 481 Table 6, an error in temperature of the EC only has little effect on the estimation of the centroid 482 position ( $\Delta l_{c}=0.6 \mathrm{~mm}$ for both coupling ducts). However, the same error in temperature in the entire 483 coupled system has more influence $\left(\Delta l_{c}=4.8 \mathrm{~mm}\right.$ for the $150 \mathrm{~mm}$ duct and $\Delta l_{c}=2.7 \mathrm{~mm}$ for the $48475 \mathrm{~mm}$ duct). The knowledge of the temperature in the EC is thus of lesser importance than that in the 485 coupling duct. 
Table 6: Influence of the temperature on the antiresonance frequency of the coupled system computed using the EA model. The influence on the centroid position estimated using the EA model is also presented.

\begin{tabular}{|c|c|c|c|}
\hline$l_{\text {duct }}[\mathrm{mm}]$ & Temperature $\left[{ }^{\circ} \mathrm{C}\right]$ & $f_{\text {antires }}^{E A}[\mathrm{~Hz}]$ & $\Delta l_{c}[\mathrm{~mm}]$ \\
\hline \multirow{7}{*}{150} & $T_{E C}=T_{\text {duct }}=19$ & 517 & 0 \\
\cline { 2 - 4 } & $T_{E C}=37, T_{\text {duct }}=19$ & 518 & +0.6 \\
\cline { 2 - 4 } & $T_{E C}=T_{\text {duct }}=37$ & 533 & +4.8 \\
\hline 75 & $T_{E C}=T_{\text {duct }}=19$ & 979 & 0 \\
\cline { 2 - 4 } & $T_{E C}=37, T_{\text {duct }}=19$ & 986 & +0.6 \\
\cline { 2 - 4 } & $T_{E C}=T_{d u c t}=37$ & 1010 & +2.7 \\
\hline
\end{tabular}

\subsubsection{Earcanal downstream section and eardrum acoustic impedance}

In previous sections, the EA model used to estimate the centroid position has accounted for both downstream and upstream sections of the EC. Since the acoustic antiresonance occurs in the EC upstream section coupled to the external duct, one may wonder if it is necessary to account for the EC downstream section and the acoustic impedance associated with the eardrum. Table 7 presents the change in centroid position estimated by the EA model when the eardrum only or the whole EC downstream section is assumed acoustically rigid. It is shown that using an infinite impedance instead of the tympanic membrane acoustic impedance implies an error of estimation in the centroid position lower than $0.2 \mathrm{~mm}$ for the $150 \mathrm{~mm}$ duct and very negligible for the $75 \mathrm{~mm}$ duct. Neglecting the entire EC downstream section has also little influence on the centroid position estimation. Therefore, this method does not require the knowledge of the tympanic membrane acoustic impedance (for an intact ear). This is good to know since the latter can be difficult to obtain with accuracy [25]. In addition, the EA model does not require the shape of the EC downstream section to estimate the centroid position. However, since the centroid position is a priori unknown before its estimation, the EC shape must be known at least up to the tympanic membrane coupling region. 
Table 7: Influence of the EC downstream section and tympanic membrane acoustic impedance on the centroid position estimated by the EA model.

\begin{tabular}{|c|c|c|}
\hline$l_{\text {duct }}[\mathrm{mm}]$ & Configuration & $\Delta l_{c}[\mathrm{~mm}]$ \\
\hline \multirow{2}{*}{150} & Rigid eardrum & -0.2 \\
\cline { 2 - 3 } & Rigid EC downstream section & -0.4 \\
\hline \multirow{2}{*}{75} & Rigid eardrum & $\approx 0$ \\
\cline { 2 - 3 } & Rigid EC downstream section & $\approx 0$ \\
\hline
\end{tabular}

\subsection{Limitations}

502 The indirect method proposed in this paper has been evaluated and investigated using a 3D FE model of a single outer ear open and occluded by an external capped duct. The FE model has been used

504 (i) to simulate the measurement and compute the antiresonance frequency from which the EA model 505 estimates the centroid position and (ii) as a reference to compare the theoretical and the centroid 506 position estimated using the EA model. The acousto-mechanical 3D FE model accounts for the 507 surrounding tissues of the EC, the external capped duct and its duct holder, and also the boneconducted stimulation. However, this model is still a simplification of a real outer ear submitted by bone-conduction. In particular, this model is truncated so the influence of the whole head vibration has not been accounted for in this study. Also, it is certain that the determination of the acoustic

511 antiresonance of the coupled system will be more difficult due to the presence of noise in measurement 512 data and to the post-treatment process, contrary to FE simulations. In addition, several sources of 513 uncertainty have been highlighted here and will affect to some extent the accuracy of the method in 514 practice. Furthermore, a stimulation by bone-transducer has been assumed here. However, the method could be used to study the influence of the type of bone-conducted stimulation on the outer ear pathway and, in particular, on the OE. Therefore, stimulation by chewing or speech could be required. However, lower jaw movement implies large deformation of the EC [41] [42]. In consequence, the position of both the coupling duct (compared to the chosen EC entrance plane) and the centroid of the EC wall 
normal vibration could vary during the measurement. In addition, incomplete seal could occur due to

520 lower jaw movement and therefore affect the accuracy of the method. Furthermore, since the same

521 bone-conducted stimulation could not be exactly obtained between open and occluded measurements

522 for chewing or speech, the acoustic pressure should be simultaneously measured in one ear open and in 523 the other occluded by the coupling duct, such as in the "real-time" OE measurement [22]. This method, 524 however, assumes a symmetry between ears (with respect to the sagittal plane) in terms of geometry 525 and bone-conducted propagation whereas differences could be expected.

\section{4. Conclusion}

In this paper, the principle of an indirect method has been proposed to estimate the centroid position of the human EC wall normal velocity induced by a bone-conducted stimulation at low frequencies $(100 \mathrm{~Hz}-1 \mathrm{kHz})$. The centroid position can be used as an indicator of the EC wall normal vibration distribution to study the influence of the bone-conduction stimulation on the outer ear pathway and on the OE. Firstly, the indirect method consists in determining the frequency of the lowest local minima of the eardrum acoustic pressure transfer function between the EC open and occluded by an external capped duct coupled to the EC entrance. These local minima correspond to acoustic antiresonances occurring in the EC coupled to the duct and are associated with sudden phase shift. Secondly, the centroid position is estimated at the lowest antiresonance frequency using an EA model associated to the coupled system in situ. The estimation consists in minimizing the difference between the antiresonance frequency measured and that estimated using the EA model. In this paper, the method has been evaluated and investigated numerically using an acousto-mechanical 3D FE model of the EC open and occluded by the external capped duct. The sensitivity of the method has been shown to increase with frequency and could be sufficient, in practice, to estimate the centroid position above $500 \mathrm{~Hz}$. To maximize the accuracy of the method, the radius of the coupling duct must be as large as possible (in the limits of the EC entrance dimension) and any incomplete seal between the duct and the 
543 EC should be avoided. Also, the EC shape, the coupling position between the EC and the duct and the

544 temperature in the coupling duct must be known as precisely as possible. On the contrary, the tympanic 545 membrane acoustic impedance is not required to estimate the centroid position in an intact ear. While 546 this study has presented the principle of the method and its numerical investigation, a future work will 547 be dedicated to its experimental application on human subjects.

\section{ACKNOWLEDGEMENTS}

549 The authors acknowledge the support of the Natural Sciences and Engineering Research Council of 550 Canada (NSERC) (funding reference number RGPIN-2016-06795). Also, the authors want to thank 551 Elliott Berger for his wise comments.

\section{APPENDIX A: MATERIAL PROPERTIES}

553 Mechanical properties of the solid domains used in the acousto-mechanical 3D FE models are given 554 in Table 8. In addition, classic values of ambient air properties are given in Table 9. It is noteworthy 555 that air properties can be calculated from the knowledge of temperature, atmospheric pressure and 556 relative humidity (see Appx. D).

Table 8: Density $\rho_{s}$, Young's modulus $E$, Poisson's ratio $v$ and structural loss factor $\eta_{s}$ of solid domains.

\begin{tabular}{|c|c|c|c|c|}
\hline Domain & $\rho_{s}\left[\mathrm{~kg} \mathrm{~m}^{-3}\right]$ & $E[\mathrm{MPa}]$ & $v[1]$ & $\eta_{s}[1]$ \\
\hline Skin & 1100 & 0.5 & 0.4 & 0.1 \\
\hline Cartilage & 1080 & 7.2 & 0.26 & 0.05 \\
\hline Bone & 1714 & 11316 & 0.3 & 0.01 \\
\hline Coupling duct & 2700 & 70000 & 0.33 & 0.01 \\
\hline Duct holder & 1050 & 0.85 & 0.48 & 0.1 \\
\hline
\end{tabular}


Table 9: Density $\rho_{0}$, sound speed $c_{0}$, dynamic viscosity $\mu$, thermal conductivity coefficient $\kappa$, ratio of specific heats $\gamma$, and heat capacity at constant pressure $C_{p}$ of ambient air.

\begin{tabular}{|c|c|c|c|}
\hline Property & Value & Property & Value \\
\hline$\rho_{0}\left(\mathrm{~kg} \mathrm{~m}^{3}\right)$ & 1.2 & $\kappa\left(\mathrm{W} \mathrm{m}^{-1} \mathrm{~K}^{-1}\right)$ & 0.025 \\
\hline$c_{0}\left(\mathrm{~m} \mathrm{~s}^{-1}\right)$ & 343 & $\gamma(1)$ & 1.4 \\
\hline$\mu(\mathrm{Pa} \mathrm{s})$ & $1.8313 \times 10^{-5}$ & $C_{p}\left(\mathrm{~J} \mathrm{~kg}^{-1} \mathrm{~K}^{-1}\right)$ & $1.0025 \times 10^{3}$ \\
\hline
\end{tabular}

\section{APPENDIX B: LOW REDUCED FREQUENCY MODEL} boundary layer thickness. In a domain $i$, the equivalent wavenumber $\hat{k}_{e q}^{i}$ and the equivalent

561 characteristic impedance $\hat{Z}_{e q}^{i}$ are given by

$$
\begin{gathered}
\hat{k}_{e q}^{i}=k_{0}\left[\frac{\gamma-\gamma-1 \psi_{t h}^{i}}{\psi_{v i}^{i}}\right]^{1 / 2}, \\
\hat{Z}_{e q}^{i}=Z_{0}\left[\psi_{v i}^{i} \gamma-\gamma-1 \psi_{t h}^{i}\right]^{-1 / 2} .
\end{gathered}
$$

In Eqs. (2) and (3), $k_{0}=\omega / c_{0}$ and $Z_{0}=\rho_{0} c_{0}$ represent respectively the free space wavenumber and characteristic impedance, $\rho_{0}$ and $c_{0}$ are respectively the air density and the sound speed, and $\gamma$

564 corresponds to the ratio of specific heats. In addition, $\psi_{v i}^{i}$ and $\psi_{t h}^{i}$ correspond to geometry and material 565 dependent functions associated to viscous and thermal effects and defined by

$$
\begin{aligned}
& \psi_{v i}^{i}=-\frac{J_{2} k_{v i} r_{i}}{J_{0} k_{v i} r_{i}}, \\
& \psi_{t h}^{i}=-\frac{J_{2} k_{t h} r_{i}}{J_{0} k_{t h} r_{i}} .
\end{aligned}
$$


In Eqs. (4) and (5), $J_{\alpha}$ is the first order Bessel function for integer $\alpha$. In addition,

$567 k_{v i}=\sqrt{-j \omega \rho_{0} / \mu}$ and $k_{t h}=\sqrt{-j \omega \rho_{0} C_{p} / \kappa}$ are the viscous and thermal wavenumbers while $\mu, C_{p}$

568 and $\kappa$ represent respectively the dynamic viscosity, the heat capacity at constant pressure and the 569 thermal conductivity coefficient. Air properties can be calculated from temperature, atmospheric 570 pressure and relative humidity (see Appx. D).

\section{APPENDIX C: COMPARISON TO EXISTING EXPERIMENTAL DATA}

572 This section provides a comparison between experimental data and simulation of the objective OE 573 induced by an external capped duct coupled to the EC entrance. Experimental data were measured by 574 Huizing on a human subject as a function of the duct length while the bone-conducted stimulation was 575 fixed at $1 \mathrm{kHz}$. Huizing used a copper duct of $500 \mathrm{~mm}$ length which was occluded at 12 different 576 positions along its length. The radius of the duct was chosen "equal" to that of the EC entrance, which 577 is a priori not circular. The seal between the EC entrance and the duct was not detailed. The bone578 conducted stimulation was ensured by a bone-transducer located at the ipsilateral mastoid. The acoustic 579 pressure was measured close to the tympanic membrane. In this work, Huizing's experiment is 580 numerically reproduced using the purely acoustical 3D FE model in order to assess the model's 581 validity. The radius of the coupling duct is equal to the equivalent radius of the EC entrance assuming 582 circular cross-section. Measured and simulated OEs induced by an external capped duct are displayed 583 in Figure 10 as a function of the duct length $l_{\text {duct }}$ (added to the EC length $l_{E C}$ and normalized by the 584 lossless wavelength calculated at $1 \mathrm{kHz}$ ). According to Figure 10, experimental and simulated OEs are 585 in relative agreement and present similar slopes which periodically decrease and increase with the 586 length of the coupling duct. 


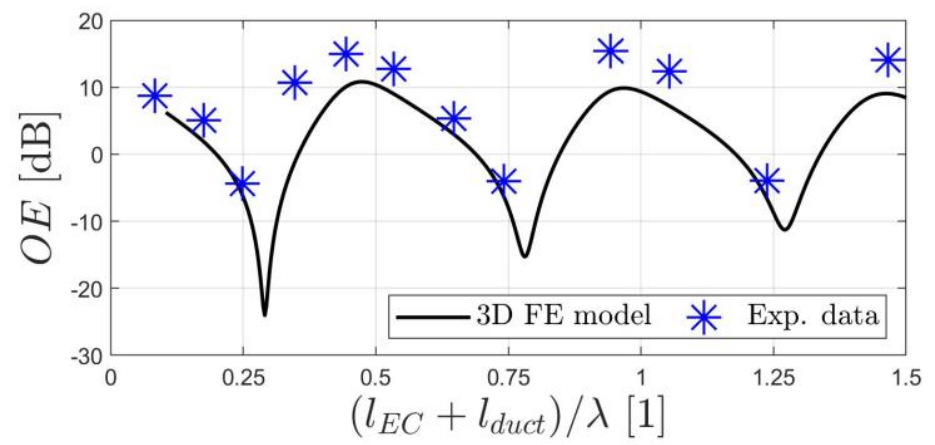

Figure 10: Objective OE induced by an external capped duct coupled at the EC entrance computed at $1 \mathrm{kHz}$ as a function of the duct length using the purely acoustical 3D FE model versus experimental data measured on a human subject by Huizing [8].

\section{APPENDIX D: CALCULATION OF AIR PROPERTIES FROM}

\section{TEMPERATURE, ATMOSPHERIC PRESSURE AND RELATIVE HUMIDITY}

This section details the computation of density $\rho_{0}$, sound speed $c_{0}$, dynamic viscosity $\mu$, heat capacity at constant pressure $C_{p}$ and ratio of specific heats $\gamma$ of air from the knowledge of the temperature $T$ (in Kelvin, $1^{\circ} \mathrm{C}$ is equal to $273.15 \mathrm{~K}$ ), atmospheric pressure $P_{a t m}$ (in Pascal) and relative

592 humidity $H_{\%}$ (in percentage). The thermal conductivity $\kappa$, however, can be assumed constant within 593 the usual range of variation of these parameters and equal to $0.025 \mathrm{~W} \mathrm{~m}^{-1} \mathrm{~K}^{-1}$.

594 The density of humid air is given by

$$
\rho_{0}=\frac{P_{a t m}}{R_{d} T}-\left(\frac{1}{R_{d}}-\frac{1}{R_{v}}\right) \frac{H_{\%}}{100} \times \frac{P_{s a t}}{T}
$$

595 where $R_{d}=287.031 \mathrm{~J} \mathrm{~kg}^{-1} \mathrm{~K}^{-1}$ and $R_{v}=461.521 \mathrm{~J} \mathrm{~kg}^{-1} \mathrm{~K}^{-1}$ are the specific gas constants for dry air and for water vapor respectively whereas $P_{\text {sat }}$ is the saturation water vapor pressure estimated as a function of the temperature [44] by 


$$
P_{\text {sat }}=0.0658 \times T^{3}-53.7558 \times T^{2}+14703.8127 \times T-1345485.0465 .
$$

598 The specific heat at constant pressure can also be estimated as a function of temperature [45] by

$$
C_{p}=4168.8 \times\left(0.249679-7.55179 \times 10^{-5} \times T+1.69194 \times 10^{-7} \times T^{2}-6.46128 \times 10^{-11} \times T^{3}\right) .
$$

600 The ratio of specific heat is then given by

$$
\gamma=\frac{C_{p}}{C_{p}-R_{d}}
$$

601 Under the ideal gas assumption, the isentropic sound speed is then calculated by

$$
c_{0}=\left[\frac{\gamma P_{a t m}}{\rho_{0}}\right]^{1 / 2} .
$$

602 Finally, the dynamic viscosity can be estimated as a function of the temperature [46] by

$$
\mu=7.72488 \times 10^{-8} \times T-5.95238 \times 10^{-11} \times T^{2}+2.71368 \times 10^{-14} \times T^{3} .
$$

\section{REFERENCES}

604 [1] E. H. Berger, L. Royster, J. Royster, D. Driscoll, and M. Layne, The noise manual, Revised Fifth edition. Fairfax, VA: AIHA Press, 2003.

[2] O. Doutres et al., "A critical review of the literature on comfort of hearing protection devices: definition of comfort and identification of its main attributes for earplug types," Int. J. Audiol., vol. 58, p. 12, 2019.

[3] S. Kochkin, "MarkeTrak VIII: Consumer satisfaction with hearing aids is slowly increasing:," Hear. J., vol. 63, no. 1, pp. 19-20, Jan. 2010, doi: 10.1097/01.HJ.0000366912.40173.76.

[4] A. Winkler, M. Latzel, and I. Holube, "Open Versus Closed Hearing-Aid Fittings: A Literature Review of Both Fitting Approaches,” Trends Hear., vol. 20, p. 233121651663174, Sep. 2016, doi: 10.1177/2331216516631741.

[5] E. Bàràny, "A contribution to the physiology of bone conduction," Acta Otolaryngol. (Stockh.), vol. 26, no. Suppl.26, pp. 1--223, 1938.

[6] G. v. Békésy, "Vibration of the head in a sound field and its role in hearing by bone conduction," J. Acoust. Soc. Am., vol. 20, no. 6, pp. 749-760, 1948.

[7] G. Békésy, "The Structure of the Middle Ear and the Hearing of One's Own Voice by Bone Conduction," J. Acoust. Soc. Am., vol. 21, no. 3, pp. 217-232, 1949.

[8] E. H. Huizing, "Bone conduction-the influence of the middle ear," Acta Oto-Laryngol, vol. Suppl. 155, pp. 1-99, 1960.

[9] J. Tonndorf, "Bone conduction. Studies in experimental animals.," Acta Oto-Laryngol, vol. Suppl. 213, pp. 1-132, 1966.

[10] S. Stenfelt, T. Wild, N. Hato, and R. L. Goode, "Factors contributing to bone conduction: the outer ear," J Acoust Soc Am, vol. 113, no. 2, pp. 902-913, 2003.

[11] S. Stenfelt, N. Hato, and R. L. Goode, "Factors contributing to bone conduction: The middle ear," J Acoust Soc Am, vol. 111, no. 2, pp. 947-959, 2002.

[12] S. Stenfelt, "Inner ear contribution to bone conduction hearing in the human," Hear. Res., vol. 329, pp. 41-51, Nov. 2015, doi: 10.1016/j.heares.2014.12.003. 
[13] I. Dobrev et al., "Sound wave propagation on the human skull surface with bone conduction stimulation," Hear. Res., vol. 355, pp. 1-13, Nov. 2017, doi: 10.1016/j.heares.2017.07.005.

[14] S. Chordekar, R. Perez, C. Adelman, H. Sohmer, and L. Kishon-Rabin, "Does hearing in response to soft-tissue stimulation involve skull vibrations? A within-subject comparison between skull vibration magnitudes and hearing thresholds," Hear. Res., vol. 364, pp. 59--67, Jul. 2018.

[15] K. Carillo, O. Doutres, and F. Sgard, "Theoretical investigation of the low frequency fundamental mechanism of the objective occlusion effect induced by bone-conducted stimulation," J. Acoust. Soc. Am., vol. 147, no. 5, pp. 34763489, May 2020, doi: 10.1121/10.0001237.

[16] J. Schroeter and C. Poesselt, "The use of acoustical test fixtures for the measurement of hearing protector attenuation. Part II : Modeling the external ear, simulating bone conduction, and comparing test fixture and real-ear data," $J$. Acoust. Soc. Am., vol. 80, no. 2, pp. 505--527, Aug. 1986.

[17] S. Stenfelt and S. Reinfeldt, "A model of the occlusion effect with bone-conducted stimulation," Int. J. Audiol., vol. 46, no. 10, pp. 595-608, 2007, doi: 10.1080/14992020701545880.

[18] M. O. Hansen, "Occlusion effects, Part II: A study of the occlusion effect mechanism and the influence of the earmould properties," Ph. D. thesis, Department of Acoustic Technology, Technical University of Denmark, Denmark, 1998.

[19] K. Lee, "Effects of earplug material, insertion depth, and measurement technique on hearing occlusion effect," PhD thesis, Virginia Polytechnic Institute and State University, Blacksburg, Virginia, 2011. [Online]. Available: https://vtechworks.lib.vt.edu/handle/10919/27021

[20] M. Brummund, F. Sgard, Y. Petit, F. Laville, and H. Nélisse, "An axisymmetric finite element model to study the earplug contribution to the bone conduction occlusion effect," Acta Acust. United Acust., vol. 101, no. 4, pp. 775-788, Jul. 2015.

[21] S. Reinfeldt, S. Stenfelt, and B. Håkansson, "Estimation of bone conduction skull transmission by hearing thresholds and ear-canal sound pressure," Hear. Res., vol. 299, pp. 19-28, May 2013, doi: 10.1016/j.heares.2013.01.023.

[22] H. Saint-Gaudens, H. Nélisse, F. Sgard, F. Laville, and O. Doutres, "Comparison of different excitations to assess the objective occlusion effect measured on human subjects," presented at the International Congress on Sound and Vibration, Montreal, QC, Canada, 2019. Accessed: Dec. 08, 2019. [Online]. Available: https://espace2.etsmtl.ca/id/eprint/19265/

[23] M. R. Stinson and B. W. Lawton, "Specification of the geometry of the human ear canal for the prediction of soundpressure level distribution," J. Acoust. Soc. Am., vol. 85, no. 6, pp. 2492-2503, Jun. 1989, doi: 10.1121/1.397744.

[24] B. L. Farmer-Fedor and R. D. Rabbitt, "Acoustic intensity, impedance and reflection coefficient in the human ear canal," J. Acoust. Soc. Am., vol. 112, no. 2, pp. 600-620, 2002, doi: 10.1121/1.1494445.

[25] H. Hudde and S. Schmidt, "Sound fields in generally shaped curved ear canals," J. Acoust. Soc. Am., vol. 125, no. 5, pp. 3146--3157, May 2009.

[26] S. E. Voss, N. J. Horton, K. E. Fairbank, L. Xia, L. R. K. Tinglin, and K. D. Girardin, "Measurements of ear-canal cross-sectional areas from live human ears with implications for wideband acoustic immittance measurements," $J$. Acoust. Soc. Am., vol. 148, no. 5, pp. 3042--3051, Nov. 2020.

[27] S. Benacchio et al., "Use of magnetic resonance image registration to estimate displacement in the human earcanal due to the insertion of in-ear devices," J. Acoust. Soc. Am., vol. 146, no. 4, pp. 2452-2465, Oct. 2019, doi: 10.1121/1.5126857.

[28] W. Lee et al., "Anthropometric analysis of 3D ear scans of Koreans and Caucasians for ear product design," Ergonomics, vol. 61, no. 11, pp. 1480-1495, Nov. 2018, doi: 10.1080/00140139.2018.1493150.

[29] H. Hudde, "Estimation of the area function of human ear canals by sound pressure measurements," J. Acoust. Soc. Am., vol. 73, no. 1, pp. 24-31, Jan. 1983, doi: 10.1121/1.388857.

[30] F. C. Karal, "The analogous acoustical impedance for discontinuities and constrictions of circular cross section," $J$. Acoust. Soc. Am., vol. 25, no. 2, pp. 327--334, Mar. 1953.

[31] F. P. Mechel, Ed., Formulas of acoustics, 2nd ed. Berlin ; New York: Springer, 2008.

[32] M. Brummund, F. Sgard, Y. Petit, and . Laville, "Three-dimensional finite element modeling of the human external ear: Simulation study of the bone conduction occlusion effect," J. Acoust. Soc. Am., vol. 135, no. 3, pp. 1433-1444, 2014.

[33] E. A. G. Shaw and M. R. Stinson, "Network concepts and energy flow in the human middle ear.," J Acoust Soc Am, vol. 69, no. 1, p. 43, 1981.

[34] E. A. G. Shaw and M. R. Stinson, "The human external and middle ear: models and concepts," in Mechanics of hearing, University Press., Delft: E. de Boer and M.A Viergever, 1983, pp. 3-10.

[35] J. Macrae and P. McAlister, "A mathematical model of acoustic leakage through air pathways past earmoulds," Aust. J. Audiol., vol. 11, no. 2, pp. 89-100, 1989.

[36] N. Atalla and F. Sgard, Finite element and boundary methods in structural acoustics and vibration. CRC Press, 2015.

[37] J. Tonndorf, “Animal experiments in bone conduction: Clinical conclusions," Trans. Am. Otol. Soc., vol. 52, pp. 22-41, 1964. 
687

688

689

690

691

692

693

694

695

696

697

698

699

700

701

702

703

704
[38] S. Schmidt and H. Hudde, "Accuracy of acoustic ear canal impedances: Finite element simulation of measurement methods using a coupling tube," J. Acoust. Soc. Am., vol. 125, no. 6, pp. 3819-3827, 2009, doi: 10.1121/1.3125344.

[39] J. Voix and L. D. Hager, "Individual fit testing of hearing protection devices," Int. J. Occup. Saf. Ergon., vol. 15, no. 2, pp. 211--219, Jan. 2009.

[40] N. Trompette, A. Kusy, and J. Ducourneau, "Suitability of commercial systems for earplug individual fit testing," Appl. Acoust., vol. 90, pp. 88--94, Apr. 2015.

[41] S. Darkner, R. Larsen, and R. R. Paulsen, "Analysis of deformation of the human ear and canal caused by mandibular movement," in Medical image computing and computer-assisted intervention: MICCAI ... International Conference on Medical Image Computing and Computer-Assisted Intervention, 2007, vol. 10, pp. 801-808.

[42] J. Bouchard-Roy, A. Delnavaz, and J. Voix, "In-ear energy harvesting: Evaluation of the power capability of the temporomandibular joint,” IEEE Sens. J., vol. 20, no. 12, pp. 6338--6345, Jun. 2020.

[43] W. R. Kampinga, Viscothermal acoustics using finite elements: analysis tools for engineers. Enschede: University of Twente [Host, 2010. Accessed: Aug. 15, 2018. [Online]. Available: http://dx.doi.org/10.3990/1.9789036530507

[44] A. D. Pierce, Acoustics: An Introduction to Its Physical Principles and Applications, 3rd ed. Springer International Publishing, 1994.

[45] Y. S. Touloukian and T. Makita, "Thermophysical properties of matter-the TPRC data series. volume 6. specific heatnonmetallic liquids and gases," Jan. 1970.

[46] D. R. Lide and H. V. Kehiaian, CRC handbook of thermophysical and thermochemical data. CRC Press, 1994. 